\title{
Characterizing Zircaloy-4 Corrosion Films Formed Under Neutron Irradiation Using Focused Ion Beam Tomography
}

\author{
Gene Lucadamo ${ }^{1}$, John Seidensticker ${ }^{1}$ and Brendan Ensor ${ }^{2}$ \\ ${ }^{1}$ Naval Nuclear Laboratory, West Mifflin, Pennsylvania, United States, ${ }^{2}$ Naval Nuclear Laboratory, \\ Schenectady, New York, United States
}

Zirconium alloys are used extensively by the nuclear industry because of their low neutron cross section and resistance to corrosion in pressurized and boiling water reactor environments [1]. Zirconium corrosion is unique because a kinetic "transition", associated with the presence of cracks and porosity in the oxide and an increasing corrosion rate, occurs repeatedly during oxidation [2,3]. Multiple phenomena are thought to further enhance the corrosion rate in-pile [4]. Focused ion beam (FIB) serial section tomography has proven to be an effective method to investigate corrosion film microstructure formed on $\mathrm{Zr}$-alloys in both autoclave [5] and reactor environments [6]. Here, the microstructure of oxide films formed on Zircaloy-4 was examined as a function of temperature, neutron flux, and neutron fluence. Test coupons were exposed in the Advanced Test Reactor (ATR) at temperatures ranging from $270-355^{\circ} \mathrm{C}$ for up to 2395 days at neutron fluxes between 0 (out-of-flux) to $1.2 \times 10^{14} \mathrm{n} / \mathrm{cm}^{2}-\mathrm{s}, \mathrm{E}>1 \mathrm{MeV}$. Oxide film thicknesses based on weight gain measurements ranged from $1.6 \mu \mathrm{m}$ (near the initial kinetic transition) to approximately $30 \mu \mathrm{m}$. Serial sectioning of the irradiated coupons was accomplished using a dual-beam, $\mathrm{Ga}^{+}$FIB-scanning electron microscope (SEM). Reconstructions consisted of up to 200 slices with a slice width of $40-50 \mathrm{~nm}$ that encompassed the entire thickness of the oxide film for a given specimen.

Differences in the oxidation behavior were apparent in one pair of specimens that were exposed to the same flux level, one at a temperature of $270^{\circ}$ and the other at $350^{\circ} \mathrm{C}$. The lower temperature specimen exhibited a significant "incubation time" where fluence accumulated without an appreciable change in corrosion rate. Cross-sectional SEM images and FIB reconstructions of the oxide revealed a wider distribution in the size of the cracks and a greater oxide-metal interface roughness in the lower temperature specimen compared to the specimen exposed at the higher temperature. The FIB reconstructions also highlighted the conformal nature of the cracks to the oxide-metal interface and embedded second phase precipitates. The measured overall porosity fractions were similar for the two specimen volumes, but the distribution of porosity through the thickness oxide film was found to depend on the exposure temperature. In particular, the oxide grown at low temperature showed coarser cracks during the incubation period and finer, closely-spaced cracks corresponding to the higher oxidation rate at high fluences, while the higher temperature specimen exhibited a more uniform distribution of larger cracks.

Quantitative measurements of oxide thickness, oxide-metal interface roughness, and porosity volume fraction were obtained from other specimens tested under different exposure conditions. Preferential oxidation along metal grain boundaries during early stages of oxidation was also observed in some specimens. The information provided from this work will be used to inform the development of a computational model for corrosion in zirconium alloys and to improve the fidelity of the model by incorporating measured microstructural parameters [7].

\section{References}

[1] AT Motta, A Couet, and RJ Comstock, Annu. Rev. Mater. Res. 45 (2015) p. 311.

[2] JS Bryner, J. Nucl. Mater., 82 (1979) p. 84. 
[3] M Gass, et al., J. Nucl. Mater., 509 (2018) p. 343.

[4] BF Kammenzind, et al., Zirconium in the Nuclear Industry: $18^{\text {th }}$ International Symposium, ASTM STP1597, RJ Comstock and AT Motta, Eds., ASTM International (2018) p. 448.

[5] N Ni, et al., Corr. Sci., 53 (2011) p. 4073.

[6] A Baris, et al., J. Nucl. Mater., 504 (2018) p. 144.

[7] The authors acknowledge Dr. Ram Bajaj (Naval Nuclear Laboratory) for helpful discussions and Dr. Arash Parsi (Westinghouse Electric Company, LLC) for performing the FIB serial sectioning and SEM imaging. 